\title{
Advanced Railway Power Quality Detecting Algorithm Using a Combined TEO and STFT Method
}

\author{
Je-Ho Yoo*, Seung-Kwon Shin**, Jong-young Park** and Soo-Hwan Cho ${ }^{\dagger}$
}

\begin{abstract}
Because an electric railway vehicle is a large scale moving load, it can cause various kinds of power quality problems in the railroad power system. The power quality impacts are considered as the strong instantaneous stresses to the related power systems and can cause an accelerating aging and a malfunction of the power supplying components. Therefore, it is necessary to detect the small and intermittent symptoms mixed in the voltage waveform. However, they cannot be detected by the triggering functions of the existing power analyzers installed in the railway systems. This paper will examine the drawback of some fast detection tools and propose an advanced detecting and analyzing method based on a combined TEO and STFT algorithm.
\end{abstract}

Keywords: Railway system, Power Quality(PQ), Detection, Teager Energy Operator(TEO), ShortTime Fourier Transform(STFT)

\section{Introduction}

A railroad system is one of the most vulnerable systems to power quality problems. Since an electric railway vehicle is considered as a large-scale concentrated load, it causes various power quality problems such as voltage sag, voltage unbalance and harmonic distortions.

So far, the common power quality(PQ) analyzer is used to deal with the power quality issues of the railways. However, the tendencies of power quality in the railway system are different from the general power supply system. For example, railroad vehicles which is a main source of electrical disturbances are intermittent and often give strong and impulse-like electrical stresses only during passing along the railway. The power supply components installed in the railway, such as transformer, insulator, circuit breaker, compensator, and so on, would be steadily deteriorated by the stresses. And, from the view point of power system, noload status and on-load status are continuously repeated.

If any component in the railway power supply system is electrically damaged and its aging progresses for a long time, the various symptoms which are small and intermittent will be contained in the voltage waveform of the idle(that is, no-load) status.

Most of the previous studies on power quality problems mainly deal with the PQ events such as voltage sag, voltage unbalance, harmonics, power factor and inrush current which are generated by the electric train [1 3].

Presently, the PQ monitoring systems are installed in the

$\dagger$ Corresponding Author: Dept. of Energy Grid, Sangmyung University, Korea. (shcho@smu.ac.kr)

* Dept. of Energy Grid, Sangmyung University, Korea. (yjh9614@sangmyung.kr)

** Metropolitan Transportation Research Center, Korea Railroad Research Institute, Korea. (skshin@krri.re.kr, jypark@krri.re.kr)

Received: June 3, 2015; Accepted: July 30, 2015 real field and used for monitoring the status of power quality of the system and the components, which is equipped with various functions of EN50160 and EN61000-4-15 standards. However, the existing PQ monitoring system is not enough to the noise-sensitive interruptions such as sag, harmonics and inter-harmonics contaminated by small noises.

Reference [4] introduces the fast fault detection technique, TEO and DESA algorithms, which have been proven to have a good and fast performance for event detection.

This paper is written and organized to examine the weakness of TEO and to introduce an advanced detecting and analyzing technique, Combined TEO(Teager Energy Operator) and STFT(Short-time Fourier Transform) algorithm. For this purpose, the current status and triggering techniques used in the railway PQ system will be reviewed at next chapter. At chapter III and IV, the combined TEO and STFT algorithm and its simulation study will be introduced. Finally, we will derive a conclusion about its usages and future applications.

\section{Status of Recent Railway PQ Monitoring System}

\subsection{General specifications}

It is roughly known that PQ analyzers have been installed at 46 substations in the Korea railway system, which can measure and record the power information such as voltage, current, real power, power factor, harmonics, PQ event and so on. And they are controlled and centralized by the GridVis system, which can manage the related data acquired by the individual analyzer devices, UMG series, of which sampling rate is $20 \mathrm{kHz}(400$ Samples/cycle for $50 \mathrm{~Hz}$ main frequency) for each 4 
channel of both voltage and current inputs. This means that 333 samples are acquired per cycle for $60 \mathrm{~Hz}$ system. And PQ analyzing functions of EN50160 and EN61000-4-15 are equipped in the device.

Main specifications of the equipment is shown as follows.

- 500MHz CPU with DSP

$-20 \mathrm{kHz}$ of sampling rate

- 50usec time window for waveform analysis

- THD analysis upto $40^{\text {th }}$ order harmonics

- Event detection for transient, overvoltage, undervoltage and overcurrent

- Communication supports for Profibus DP V0, Modbus RTU, Modbus TCP/IP, Modbus Gateway and BACnet.

\subsection{Event detection techniques}

Because most of PQ events in the power supply system occur intermittently and instantaneously, the detection techniques which can sort them from the enormous data acquired by the DAQ devices are very essential. And the limited storage capacity of PQ digital device makes the techniques, also known as triggering, more important.

The triggering conditions generally used in the PQ analyzers are as follows:

- Upper or Lower level trigger: This is the most common method generally used for the RMS(root-mean-square) based event triggering, such as voltage sag, swell. Every RMS value of voltage, current or other power values is compared with the upper or lower limits which have already been set according to the related IEC or the national standards.

- RoC(Rate of Change) trigger: The trigger is initiated when the change rate of the successive measurement data of voltage, current, active power, reactive power and frequency during 1 -cycle exceeds $2 \%$, which is changeable.

- Power swing trigger: The trigger is initiated when the measured active and reactive powers fluctuate or swing over the fixed limit.

- Event trigger: This triggering function uses the binary contact inputs, 0 or 1 , by which the occurrence of an event can be indicated.

Generally, the upper or lower level trigger or the event trigger are used for the voltage and current RMS-based PQ event detections. But this triggering method is not enough to detect the instantaneous anomalies added in the voltage and current signals of railroad power system.

\section{Combined TEO and STFT Algorithm}

The combined TEO(Teager Energy Operator) and STFT(Short-Time Fourier Transform) algorithm is devised for the fast detection and the detail analysis of small-size voltage event signals containing a good information on the defective and aging status.

\subsection{Teager Energy Operator(TEO)}

The TEO algorithm is a non-linear operator which can be implemented in the real time PQ event detection, owing to the very fast time response [3]. And it estimates the pattern of sinusoids by using only 3 consecutive data under the assumption that the analyzed signal is a purely sinusoidal waveform. This inherent characteristic makes it used in many applications about PQ monitoring, especially, transient phenomenon and voltage fluctuation or flicker [5] [7]. Unlike the previous papers, the reference [4] deals with an applicability of TEO and discrete energy separation algorithm(DESA) for waveform distortions like harmonics, inter-harmonics and frequency variation. However, most of them are focusing on the detection and the data extraction by DESA, leading to a result that the simple TEO method has an excellent performance to detect the starting and ending instances of an event.

The value of TEO, $\Psi[\mathrm{x}(\mathrm{t})]$, of an oscillating continuous signal, $x(t)=A \cos \left(\omega_{c} t+\theta\right)$, is calculated as follows:

$$
\Psi[x(t)]=[\dot{x}(t)]^{2}-x(t) \ddot{x}(t)=A^{2} \omega_{c}^{2}
$$

where

$$
\begin{gathered}
x(t)=A \cos \left(\omega_{c} t+\theta\right) \\
\dot{x}(t)=-A \omega_{c} \sin \left(\omega_{c} t+\theta\right) \\
\ddot{x}(t)=-A \omega_{c}^{2} \cos \left(\omega_{c} t+\theta\right)
\end{gathered}
$$

Eq. (1) can be converted into the equivalent discrete version as shown below.

$$
\Psi[x[n]]=[x[n]]^{2}-x[n-1] x[n+1]
$$

This equation shows that values of TEO are calculated with 3 consecutive time data.

However, such properties of TEO can act as a drawback at the same time advantage. It is impossible to distinguish whether the irregularity of TEO is caused by interharmonics or small noise, because the TEO estimates a sinusoid with only 3 consecutive points. In other words, the TEO is very weak at the noise components not filtered out, because it confuses the noise signal mixed in the 3 points with the instantaneous sinusoidal component.

Figs. 1 and 2 show the TEO analysis results of 10\%, 4 cycle voltage sag signal without and with a small noise. Additionally, the magnitude and frequency components are restored by DESA(Discrete Energy Separation Algorithm) as referred to the reference [4].

From the Fig. 2, we have a conclusion that even small noise $(0.03 \%$ noise corresponds to $0.066 \mathrm{Vrms})$ can cause a 

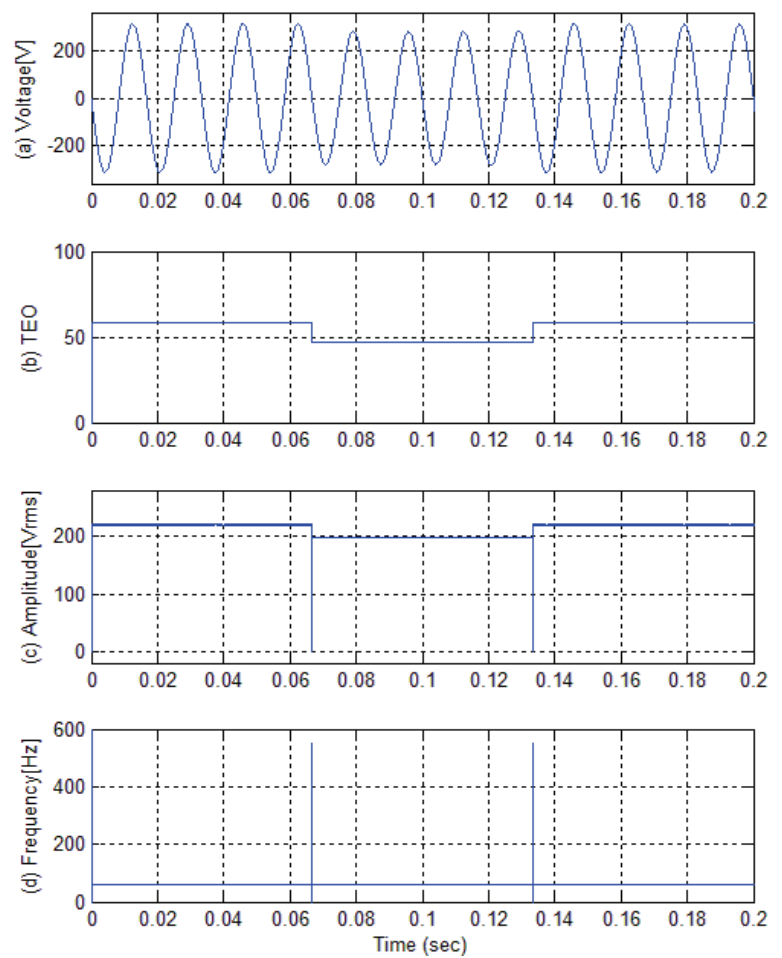

Fig. 1. (a) 8\%, 4-cycle voltage sag signal without a noise; (b) its TEO analysis; (c) the amplitude restoration and (d) the frequency restoration by DESA.
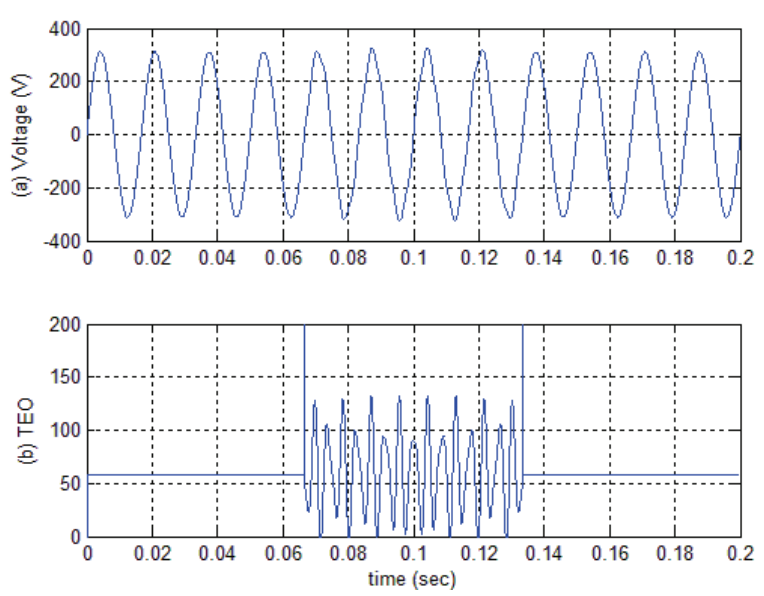

Fig. 3. (a) voltage waveform containing $5 \%, 290 \mathrm{~Hz}$ interharmonics without a noise and (b) its TEO analysis.

large distortion of the TEO values, which is more clear from the DESA analysis.

This phenomenon leads to more severe distortion to the case of inter-harmonics. Figs. 3 and 4 show the TEO analysis result of $5 \%, 290 \mathrm{~Hz}$ inter-harmonics without and with a small noise.

And Figs. 5 and 6 show the TEO analysis result of $1 \%$ periodic notches of $0.0833 \mathrm{msec}$ duration without and with a small noise. From these figures, we can have a conclusion that the TEO analysis can recognize the notches very sensitively, while we cannot recognize the presences
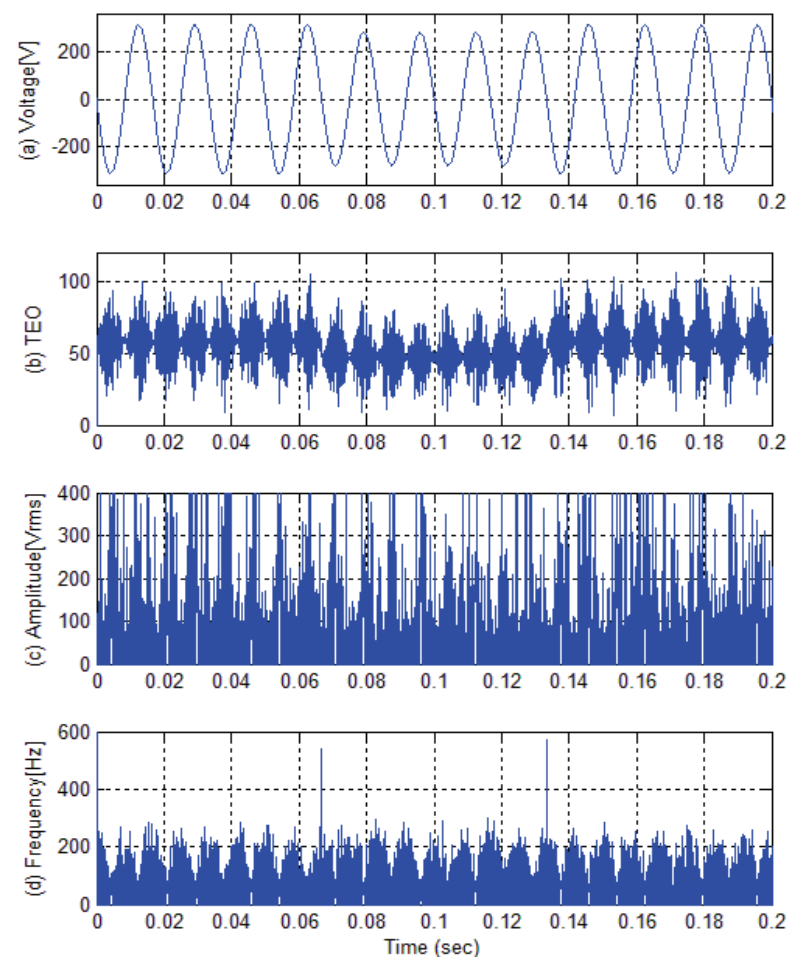

Fig. 2. (a) $8 \%$, 4-cycle voltage sag signal with a $0.05 \%$ noise; (b) its TEO analysis; (c) the amplitude restoration and (d) the frequency restoration by DESA.
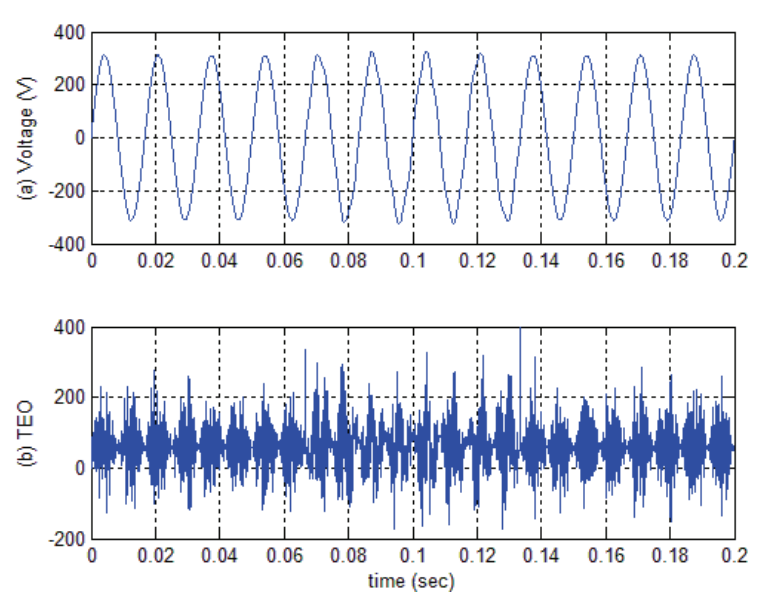

Fig. 4. (a) $5 \%, 290 \mathrm{~Hz}$ inter-harmonics voltage signal with a $0.05 \%$ noise and (b) its TEO analysis.

of notches and noises in the voltage waveform. Because the TEO regards the notches as a kind of noises, it can distinguish the magnitude of notch from the different noises.

By seeing the Figs. 5 and 6, we can make a conclusion that the TEO shows a very excellent performance to fast detect the instantaneous abnormal signals while they cannot be recognized by the simple threshold setting and the RMS-based calculation in which the spike-like anomalies get buried under the surrounded sinusoidal waveforms. 

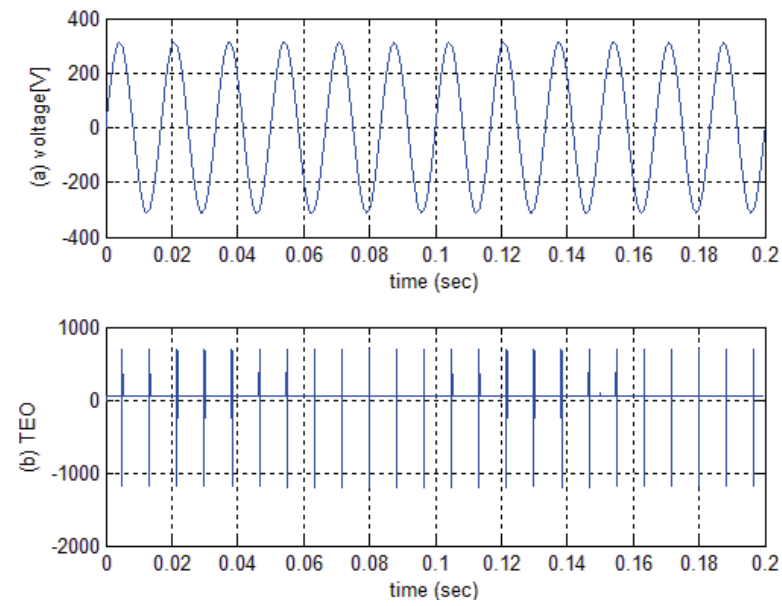

Fig. 5. (a) voltage waveform containing $1 \%$, periodic notches of $0.0833 \mathrm{msec}$ duration without a noise and (b) its TEO analysis.
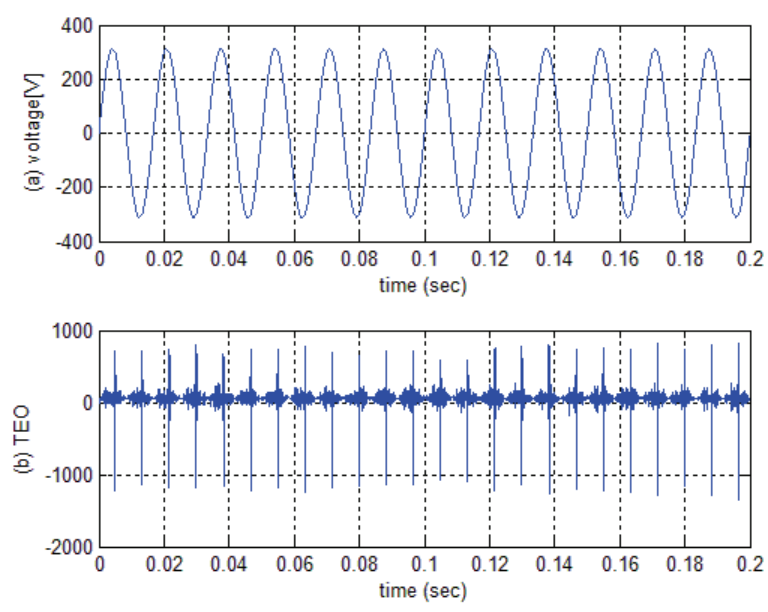

Fig. 6. (a) voltage waveform containing $1 \%$, periodic notches of $0.0833 \mathrm{msec}$ duration with a $0.05 \%$ noise and (b) its TEO analysis.

The noise considered in this study is dependent on the Nyquist frequency and not filtered out by the low pass filter(LPF) located in the front terminal of the data acquisition(DAQ) unit.

\subsection{Combined TEO \& STFT algorithm}

In order to overcome the drawback of TEO mentioned in the previous section, we employed the short-time Fourier transform(STFT) and would like to propose a new and advanced detection algorithm, the combined TEO and STFT algorithm.

As one of the time-frequency analysis method, the shorttime Fourier transform(STFT) is used to analyze the sinusoidal frequency and phase contents in some sections of a continuous time series signal.

A continuous-time STFT is a Fourier transform using a sliding time-window and can be written as:

$$
\begin{aligned}
\operatorname{STFT}\{x(t)\}(\tau, \omega) & \equiv X(\tau, \omega) \\
& =\int_{-\infty}^{\infty} x(t) w(t-\tau) e^{-j \omega t} d t
\end{aligned}
$$

where $\mathrm{w}(\mathrm{t})$ is a time-window function and $X(\tau, \omega)$ is simply the Fourier transform of $x(t) w(t-\tau)$.

To implement the STFT in the digital domain, its equivalent discrete version is needed, which is called as discrete-time STFT. It is defined as follows:

$$
\begin{aligned}
\operatorname{STFT}\{x[n]\}(n, \omega) \equiv & X(n, \omega) \\
& =\sum_{k=-\infty}^{\infty} x[n] w[k-n] e^{-j \omega k}
\end{aligned}
$$

After the TEO values are obtained, the values will be processed to calculate their frequency component by using the STFT with a window size of 256 samples. In this analysis, it is impossible to convert the TEO to the exact frequency domain values because the TEO values are basically considered as a constant in the energy dimension of the voltage signal. Therefore, the variation of the magnitudes of TEO will be shown as a DC component.

\section{Simulations}

In this part, the simulation results for the previous cases of Fig. 2 and Fig. 4 will be shown. Only with the TEO analysis, it is impossible to accurately detect the starting and ending instants in the waveform contaminated with a noise.

Fig. 7 shows the simulation result of the combined TEO $\&$ STFT analysis of the $8 \%$, 4-cycle voltage sag signal with a $0.05 \%$ noise. Compared with the Fig. 2-(b), the small event is easily recognized. And it can be found out that the noise components are widely distributed in higher order frequency region.

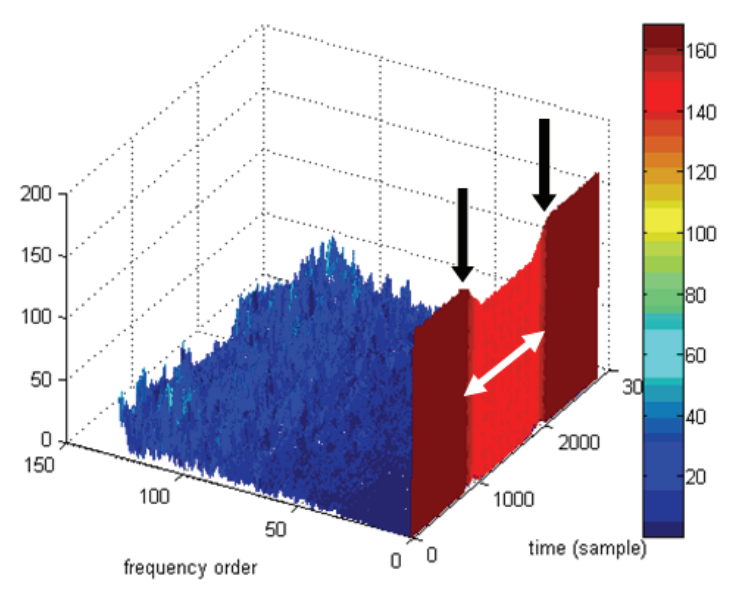

Fig. 7. Result of Combined TEO \& STFT analysis for the same voltage signal as Fig. 2 

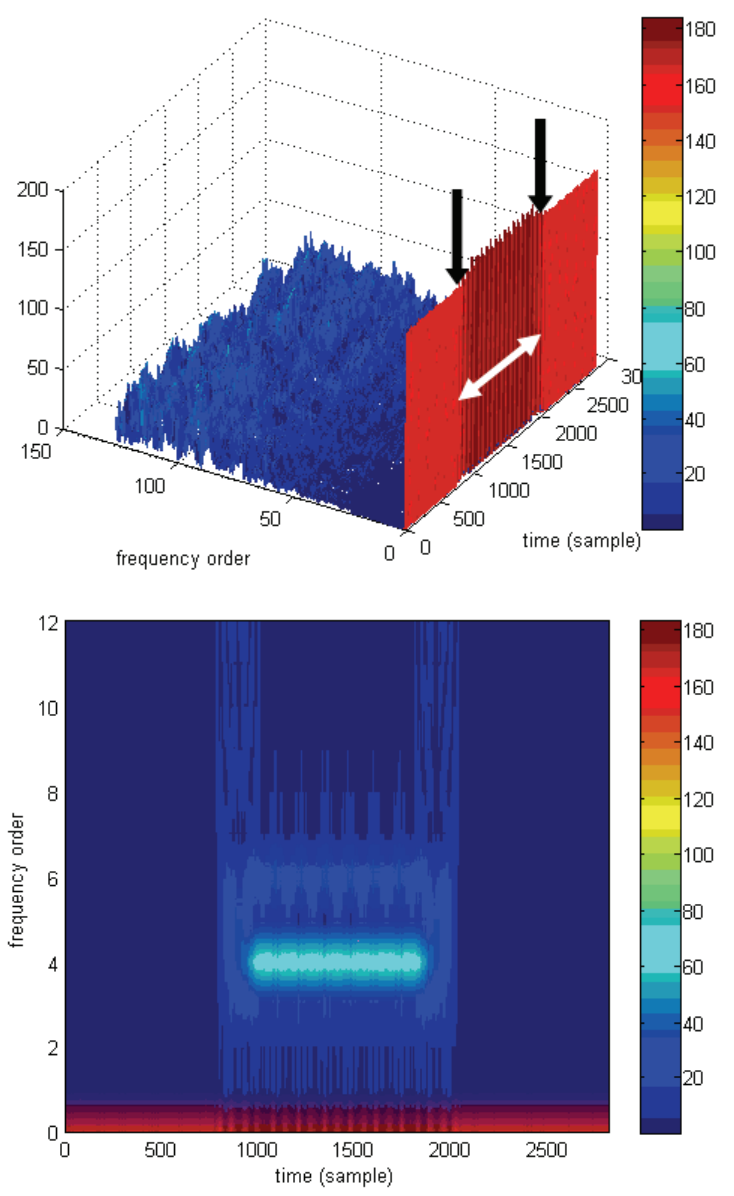

Fig. 8. (top) Result of Combined TEO \& STFT analysis for the same voltage signal as Fig. 3, (bottom) 2Dgraph

Fig. 8-(top) shows the simulation result of the combined TEO \& STFT analysis of the $5 \%, 290 \mathrm{~Hz}$ inter-harmonics voltage signal with a $0.05 \%$ noise. Compared with the Fig. 3-(b), the small event is easily recognized in the DC region.

Additionally, we have considered the multiple harmonics voltage signal in order to compare the previous interharmonics case.

In the Fig. 9, a voltage signal containing the multiple harmonics of $3^{\text {rd }}, 5^{\text {th }}, 7^{\text {th }}$ and $9^{\text {th }}$ components less than $3 \%$, was analyzed by the combined TEO and STFT method. Difference between inter-harmonics and harmonics is not obvious in the 3D-graph of combined TEO \& STFT analysis. However, the difference becomes clear from the $2 \mathrm{D}$-graphic analysis. For the harmonics, the distribution of magnitude in the frequency domain is in the regular pattern.

\section{Conclusion}

Teager Energy operator(TEO) is a good and simple analysis method which can detect some interruptions of the
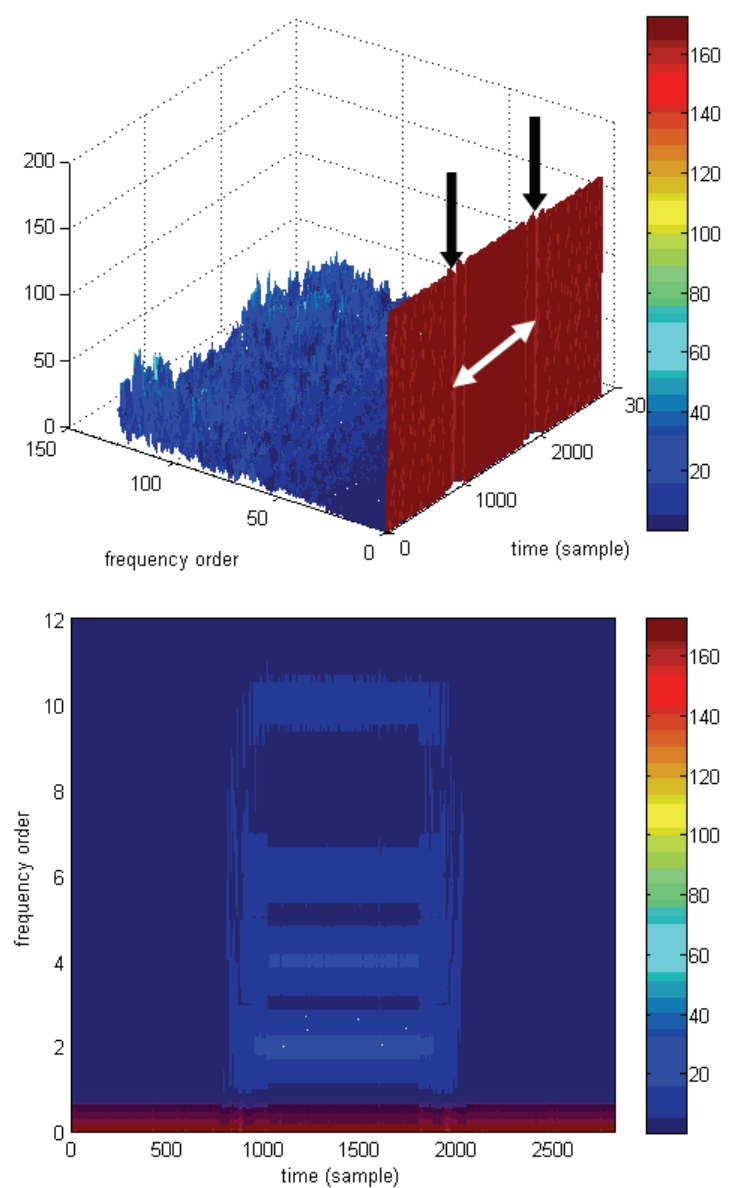

Fig. 9. (top) Result of Combined TEO \& STFT analysis of the harmonics voltage signal, (bottom) 2D-graph

voltage signal very fast. However, it has a drawback that the TEO is very weak for the noise environment. In order to make up for its defect, a new and innovative analysis method of the combined TEO and STFT algorithm is presented in this paper. It is shown that the proposed method has a good detecting performance under the noise contamination.

Now, it is expected that the combined TEO and STFT method is a good application of power quality analyzers installed in the railways to recognize the small event signals. By the method, an aging status of railway power systems and the related system components will be estimated in real time. Furthermore, it can be also applied to the power quality-based predictive maintenance (PQPM) or reliability centered maintenance (PQRCM) of power supply units installed in the railway power system.

\section{Acknowledgements}

This research was supported by a grant from R\&D Program of the Korea Railroad Research Institute, Republic of Korea. 


\section{References}

[1] H. M. Lee, G. D. Kim, S. C. Oh and C. M. Lee, "Optimal Design for Power Quality of Electric Railway," Energy Conversion and Management, vol. 52, Iss. 4, pp. 1959-1967, 2011.

[2] K.C. Kim, S.E. Jin, J.H. Lee and Y.S. Jeon, "Timevarying Harmonics Measurement and Evaluation of the High Speed Electric Train Loads," Journal of the Korean Institute of Illuminating and Electrical Installation Engineers, vol. 20, no. 7, pp. 29-37, 2006.

[3] A. Bubasi, A.S. Yilmaz and K. Tufan, "Detection of Generated and Measured Transient Power Quality Events Using Teager Energy Operator," Energy Conversion and Management, vol. 52, Iss. 4, pp. 1959-1967, 2011.

[4] S.H. Cho, J. Hur and I.Y. Chung, "An Applicability of Teager Energy operator and Energy Separation Algorithm for Waveform Distortion Analysis: Harmonics, Inter-harmonics and Frequency Variation," The Journal of Electrical Engineering \& Technology, vol. 9, no. 4, pp. 1210-1214, 2014.

[5] M.A. Eldery, E.F. El-Saadany and M.M.A. Salama, "A Simple Energy Operator Computational Method for Voltage Flicker Assessment," IEEE Transactions on Power Delivery, vol. 21, no. 3, pp. 1743-1750, 2006.

[6] T. K. Abdel-Galil, E. F. El-Saadany and M. M. A. Salama, "Online Tracking of Voltage Flicker Utilizing Energy Operator and Hilbert Transform," IEEE Transactions on Power Delivery, vol. 19, no. 2, pp. 861-867, 2006.

[7] S.H. Cho, J.U. Kim and J.H. Han, "Determination of Power-Quality Disturbances Using Teager Energy Operator and Kalman Filter Algorithms," International Journal of Fuzzy Logic and Intelligent Systems, vol. 11, no. 2, pp. 1959-1967, 2011.

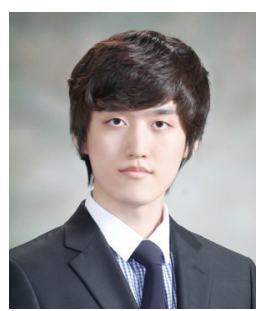

Je-Ho Yoo received his B.S. degrees in Dept. of Energy Grid from Sangmyung University, Seoul, Korea, in 2014. Currently, he is pursuing M.S. degree in Dept. of Energy Grid, Sangmyung University. His research interests include power quality analysis including measurement, detection and classifycation and signal processing techniques for PQ analysis.

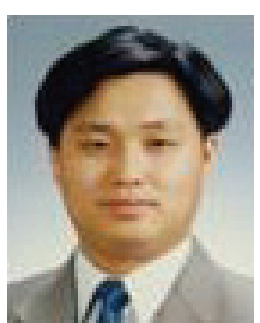

Seung-Kwon Shin received his B.S., M.S. degree in in Electrical Engineering and $\mathrm{Ph}$. D degree in School of Electrical and Computer Engineering from Sungkyun-kwan University, Korea, in 1995, 1998 and 2001, respectively. Currently, he is a Senior Researcher in Korea Railroad Research Institute (KRRI). His research interests include a power electronic control and a railway substation including power quality, measurement and detection.

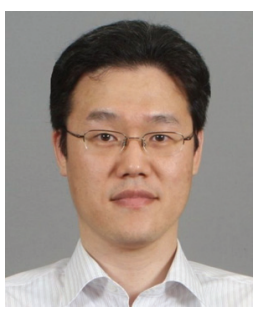

Jong-young Park received the B.S., M.S., and Ph.D. degrees from Seoul National University, Seoul, Korea, in 1999, 2001, and 2007, respectively. He was a Senior Researcher at LSIS Co., Ltd., Korea from 2009 to 2013. Currently, he is a Senior Researcher at Korea Railroad Research Institute (KRRI). His recent research interests include the optimal operation of power systems in railway with the smart grid technology.

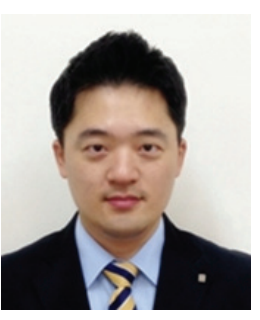

Soo-Hwan Cho received his B.S., M.S. and Ph.D. degrees in Electrical Engineering from Korea University, Seoul, Korea, in 2002, 2006 and 2009, respectively. He was an Engineer at Samsung Electronics from 2002 to 2004 and a Senior Researcher at Korea Atomic Energy Research Institute (KAERI) from 2009 to 2011. Currently, he is an assistant professor in the Department of Energy Grid, Sangmyung University, Seoul, Korea. His research interests include power quality analysis including measurement, detection and classification and signal processing techniques for PQ analysis. 